\title{
Computing Pattern in Pervasive Healthcare
}

\author{
Sriram R, \\ PG Scholar, \\ Dept. of CSE, \\ Sri ManakulaVinayagar \\ Engineering College
}

\author{
Madhusudanan J, \\ Ph.D \\ Associate Professor, \\ Dept. of CSE, \\ Sri ManakulaVinayagar \\ Engineering College
}

\author{
Prasanna \\ Venkatesan V, Ph.D \\ Associate Professor, \\ Dept. of Banking \\ Technology, \\ Pondicherry University
}

\author{
Geetha S, \\ Research Scholar, \\ Dept. of Banking \\ Technology, \\ Pondicherry University
}

\begin{abstract}
The Pervasive or Ubiquitous computing is the embedded technologies which involve computing and communication capability, which seamlessly integrated with the end user. The concept of pervasive computing composed of Artificial Intelligence, Human Computing Interface and Context-aware Computing which makes computing to appear everywhere and anywhere. Many research is going in Pervasive computing in that healthcare is one of the field which is fast growing. The pervasive computing and healthcare is combined and known as Pervasive Healthcare. It address those technologies and concept that integrate healthcare more seamlessly to our everyday life. In this project, a framework using the architectural pattern such as pipe and filter pattern and model view controller pattern is proposed. The framework will provide intricate monitoring, and probabilistic and filter algorithm to take the decision accurately.
\end{abstract}

\section{General Terms}

Pipe and filter architectural pattern and Model View Controller architectural pattern.

\section{Keywords}

Pervasive computing, Pervasive healthcare, Architectural pattern, Context aware.

\section{INTRODUCTION}

The Ubiquitous computing refers to the concept like disappearing computer, "when they are everywhere and anywhere," and pervasive computing means integration of computing power and sensing into anything, including not only computers, personal digital assistants, etc.,. The Pervasive Computing is the embedded technologies which involve computing and communication capability, which seamlessly integrated with the end user [1]. Pervasive computing touches on a wide range of research topics includes distributed computing, mobile computing, contextaware computing and many. This research is going to concentrate on the Context aware computing and it is the component of pervasive computing. It is used to sense and react according to the environment. The context-awareness is done in many fields to make the process simple and efficient. The adoption of context-aware services in healthcare lagging behind due to significantly alter the workflow pattern to accommodate the system. This also gives the simplicity, efficiency, accuracy and performance in the healthcare service. The pervasive computing in healthcare is combined, known as Pervasive Healthcare [2]. The pervasive healthcare making the healthcare available everywhere, anytime. It address those technologies and concepts that integrate healthcare more seamlessly to our everyday life, wherever we are. The proposed work evaluates and analyses the contextaware framework using Colored Petri net tools in real-life setting. This also gives the intricate monitoring and probabilistic relevance and filter algorithm. The Architectural patterns are used to make the context-aware framework more feasible and scalable. The architectural pattern such as pipe and filter and model view controller are used in this framework.

The technology is moving beyond the personal computer to everyday device with embedded technology and connectivity as computing devices become progressively smaller, more powerful and existing everywhere. Pervasive Computing is growing trend towards embedding technologies in everyday objects, so they can communicate with the devices which are completely connected and constantly available. The Contextaware computing is the component of pervasive computing. It can sense their environment and react according to their behavior. Many Researches are done and going on in various field which are more attractive and interesting. The research in healthcare is trending in pervasive computing, by combining this it known as pervasive healthcare, though healthcare service is available everywhere and anytime. The pervasive healthcare addresses those technologies and concepts that integrate healthcare more seamlessly to our everyday life, wherever we are.

\section{RELATED WORKS}

The review is done with respect to pervasive healthcare and patterns used in it. The Pervasive healthcare comes under the domain of pervasive computing and we also use context aware computing. We have reviewed many paper on these basis, to know the related work. Some of the related works are discussed and with this we provide a proposed system.

The Self-learning Context-aware framework is the framework which we take as a base to generate over proposed framework [4]. The framework gives the layers which includes devices, sensor and software to deliver context information. The layer in the framework are context provider layer, semantic reasoning layer, knowledge query layer and persistence layer, these four layers are come under the context aware platform, with that there is learning engine, it consist of data collection module, input converter, pre-processor, data mining, postprocessor, decision module and integration module.

The context provider layer gets the time, location, environment and other context information. These information are passed to the semantic reasoning layer, it consist of knowledge base and reasoning base. The information first passed to the knowledge base, there the relevant context information is changed into formal context known as ontologies [11]. The context aware information and existing medical details are integrated into the platform. The description logic reasoner in the reasoning base provides the reason with the help of rule based reasoning. It also detects various modules and gather all context information from 
corresponding data, these knowledge is used in different services and application by retrieving context information. The persistence layer stores the context information and gives awareness according to the changing requirement and behavior of the user, such as previous location of caregiver and action or patient taken by the users. It derive pattern and trend to adapt information from the learning engine which exploits history of context information.

The context aware platform is based on the ontology based platform and context aware. All devices and the software are included in Device layer that deliver context information. The modern healthcare has many computerized medical equipment to deliver the patient condition, e.g., electronic patient records, monitoring equipment, lab result and supports the patient in daily activities. The pipe and filter architectural pattern is used to design the learning engine. In this pattern the larger task is processed into a smaller independent processing sequence called filters, which are connected by channels called pipes. Using this the architecture becomes very modular, reusable, extensible and flexible. The input conversion, data collection module, integration module and data mining present in this layer. Learning engine consists of pre-processor, data mining, post-processors and decision module. These helps to learn new context and take decision according to the information of context.

The next related framework is Context Aware Real Time Assistant System [6,12], this designed to gather data with provided context information and helps to take the decision in healthcare service. The framework is achieved by fuzzy rule based reasoning and case based reasoning [3]. The context information is gather from the sensors are processed and integrated to produce the fuzzy sets and queries. The fuzzy rules are used to get medical condition and to identify current situation of the user. The case retrieval done by retrieving similar care and fuzzy output. If it is new case, gives alarm, collect data and store the case. A fuzzy system consists of three main parts they are fuzzy set, rules and inference engine. The fuzzification is a crisp set of input data gathered and converted to a fuzzy set using fuzzy linguistic variable, terms and membership function. The resulting fuzzy output is mapped to crisp output using membership in the defuzzification.

The case based reasoning is a model for combining problem solving and learning, it retrieve already solved problem similar to current one and attempt to modify solution to fit for current problem. The case based reasoning application can be described by a cycle composed of retrieve similar case, revise the solution, retain the experience to use in future for problem solving and reuse the information and knowledge.

The ubiquitous context aware healthcare service is the framework which gives the medical service through semantic web by combining the application of SOA, this supports user at the time of complex problem [5]. The aim of this framework is to locate the services automatically according to the functionalities of web services. The semantic web is discovered with the help of UDDI, and it provides business entities, binding templates, service entities to represent detail of service and business. This framework composed of healthy life mapping, situation aware of medical tourism, intelligent healthful food decision and emergency indication and ambulance dispatch. The Ubiquitous context aware healthcare service is formed by integrating these four services. Through the nature medicine service, it provides the information on tour, nearby hospital and medical services, restaurant and decision for healthy food.
The architectural pattern can be used in pervasive healthcare with the help of context-aware. There are many architectural pattern, the Model View Controller architectural pattern is used with context in related work [7]. The idea of this pattern is that each component should be independent of other, so the changes done in one component do not constrain programmer to make change in other. This makes the easy way to employ contextual part from various components [10]. The model gets each request for a change or receive data and it is responsible for storing and managing data with the help of DBMS. The controller host the application codes and handles relation with other component. It receives a user request from view and prepare suitable response with help of model and context component. It also displays result by manipulating with view. The context component in mode view controller is responsible for communication to context management system, defining situation from high-level context, modelling context data to unified format and presenting situation to other components. The controller should able to send information to context component and directly send to context management system. The context information helps context component to make exact situation and view it.

A fuzzy-based Context Reasoning system is other framework [8] which delivers the healthcare with possible innovative through internet technology, which facilitate secure and efficient sharing of clinical data and patient. The patient details are monitored by different kind of sensors and it deployed. The rule engine or reasoning plays a crucial role in system both on gateway and server side. It can be personalized with different rules for different application and it also executes real time and offer notification of critical condition [9]. The reasoning component is capable of performing three main task they are continuous context of physical state, prediction of risky situation and emergency situation notification. These are the related work reviewed, with the help of these framework, we have planned to develop the proposed framework.

\section{EXISTING SYSTEM}

The framework is introduced to develop a self-learning the new context given by the user, using rules and other ontologies given in the framework. The self-learning of the context is done through the learning engine. The information is then collected by algorithm that identify missing or inaccurate knowledge in the context-aware platform. These information are filtered, cleaned and structured to give input for data mining and as a result, it gives prioritized and filtered data by associating probabilistic. This self-learning and probabilistic framework works to adapt the behavior of ontology-based, context-aware application to the changing requirement of the user and their workflow pattern. The probabilistic approach conveys the reliability of the learned knowledge to the users and ensures the compatibility with existing knowledge in context model. This framework is used for simple monitoring of the patient and alerting or beeping for the nurse call.

The limitation of existing framework, learns only the context and recognizes new context with the information given to it. This is not analyzed and evaluated in the formal method. The complex monitoring is not done in this framework. The crisp output is not send to the doctor or guardian in time of emergency. It doesn't support patient in out hospital condition. 


\section{PROPOSED SYSTEM}

The Context-aware framework is dynamic, so it can apply to different services, domains and different environment. This work is to verify the behavioral properties of the contextaware framework in a healthcare system. The Context-aware framework which does the self-learning of context with the given rules and ontology, by gathering data from current and existing locations. It gives the healthcare service to learn the new context of its own and behave according to the user. This system does only self-learning process of context in healthcare system and used to monitor the patient through it. The learning engine is used to just learn the new context and it doesn't use for learning other details. This framework doesn't give the complex monitoring system, and the self-learning context probabilities is adapted by the increase or decrease of context and behavioral information gathered about the usage of the learned information. This is not deployed and evaluated in a real-life healthcare setting.

\subsection{Architectural Pattern}

In the proposed work we use two architectural pattern, they are pipe and filter and model view architectural pattern. The pipe and filter architectural pattern provides a structure for systems that process a stream of data. Each processing step is encapsulated in a filter component and data are passed through pipes between adjacent filters. The filter transforms or filters the data it receives via the pipes with which it is connected. A filter can have any number of input pipes and any number of output pipes. The pipe is the connector that passes data from one filter to the next. It is a directional stream of data that is usually implemented by a data buffer to store all data, until the next filter has time to process it. Model View Controller is a design pattern used in services architecture. It is a software design pattern for developing web applications. The Model is the lowest level of the pattern which is responsible for maintaining data. View is responsible for displaying all or a portion of the data to the user. Controller is software code that controls the interactions between the model and view.

\subsection{Advantage}

The advantage of the proposed system gives the framework which gives intricate monitoring. The framework has been verified and analyzed in a healthcare environment using the tools. This also provides support for the patient in mobile and good alerting system at the time of emergency. The architectural pattern used in this framework will provide many features like scalability, feasibility and so on. It allows to take decision making correctly by comparing with the historical information provided in the framework.

\section{RESEARCH CONTRIBUTION}

The context-aware framework is evaluated and deployed according to the real-life setting in the smart environment. This framework will provide more intricate monitoring and probabilistic relevance and filter algorithm. The Architectural patterns are used to make the context-aware framework more feasible and scalable. The architectural pattern such as pipe and filter, model view controller and behavioral patterns are used in the proposed framework. The comparison of similar case is done and decision is provided in an effective way. The historical information of both patient and doctor is gathered for the verification when in need. This framework gives good alerting system by beeping to nurse, it also send a crisp report to the doctor and guardian about the patient status. Through this framework we also propose to give support for the stakeholder in out hospital condition also.

\section{CONTEXT-AWARE COMPUTING PATTERN}

The context aware computing pattern is the framework which gives the real time setting of the hospital. The context aware computing pattern has various components which makes to provide efficient framework, they are patient context source, service manager, information enquiry, monitoring level, data pipeline, data manager and integration module.In this patter the source given as patient context, these context given to the service manager. With the help of data manager it passes to the data pipeline and integrate with data manager through integration module. The information is gathered from information enquiry.

The patient context source is the component where the context of every patient is taken as input and given to the service manager. The service manager consists of following components they are Patient, Doctor and Type of Disease. In the service manager, the collected patient context are gathered and stored as patient details. In a healthcare, $\mathrm{n}$ number of patient will come to consult doctor or to cure disease, so each patient details are stored separately. The service manager manages the $n$ patient by type of disease with the help of rules and medical ontology in data manager and identifies the respective doctor to consult. The service manager then leads to data pipeline and information enquiry.

The data pipeline gets the input from the service manager and it consists of Data Orchestration, Data Mining, Data Processing and Decision Making. The data pipeline is done by pipe and filter architectural pattern. The Data orchestration collects the details of every patient and order each one with respect to the type of disease and the respective doctor. The ordered details are forwarded to the data mining, where the patient current and previous medical details are mined.

This will be helpful for taking the decision rightful way, through the data mining we can also look over the similar case for verification and also we can integrate new case to data manager through integration module. The mined data are send to data processing, where the evaluation of patient is done by the doctor with the help of available data and decision is made through decision making.

The integration module is to integrate the new case to the knowledge database. The information enquiry gives the details of patient admitted in the hospital and also give about the monitoring level such as general and intricate. The general is the ordinary monitoring and intricate is the complex monitoring. The monitoring level is done by use of the model view controller architectural pattern.

The data manager consists of two components they are Rules and medical ontology, and knowledge database. It is used to coordinate with the service manager and data pipeline. The patient, type of disease and doctor in the service manager are coordinated with the rules and medical ontology in data manager. The knowledge database used to store the new and similar case with the patient details 


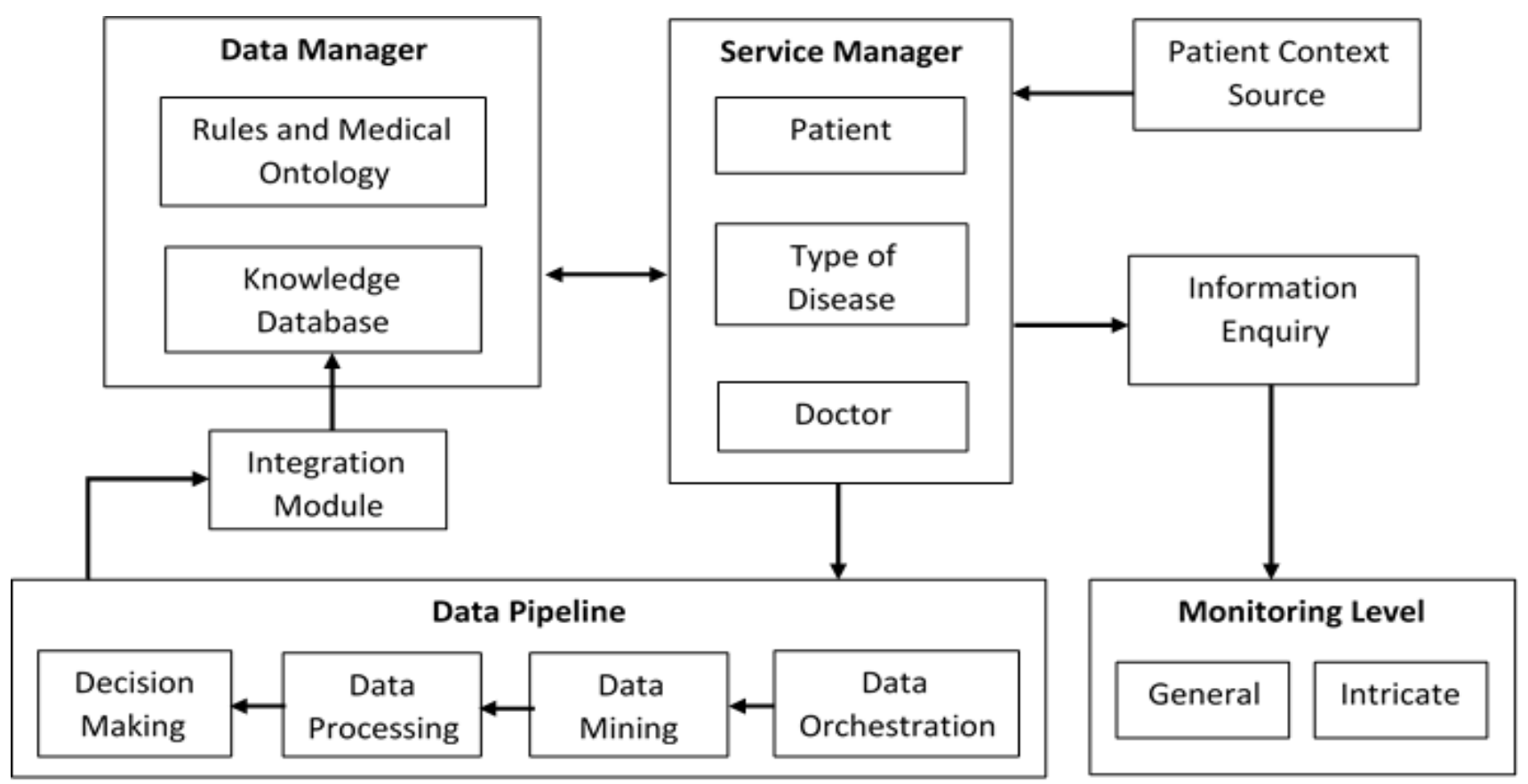

Fig 1: Architecture Diagram of Context-aware Computing Pattern framework

\section{CONCLUSION}

The Healthcare is made available everywhere and anytime using the technologies of Pervasive Computing. The contextaware computing is the component of pervasive computing, through which the healthcare framework is created using patterns. The Pipe and Filter Pattern and Model View Controller Context Pattern is used in this framework. The hybrid context aware computing pattern framework is designed which provide the intricate monitoring and takes decision with the help of probabilistic and filter algorithm. In future we can evaluate and analysis with the appropriate tool to prove the framework efficient in real life setting using tools.

\section{REFERENCES}

[1] M. Satyanarayanan., "Pervasive Computing: Vision and Challenges", to appear in IEEE Personal Communications, 2001.

[2] IlkkaKorhonen, Jakob E. Bardram., "Guest Editorial Introduction to the Special Section of Pervasive Healthcare," IEEE Transactions on Information Technology in Biomedicine, vol. 8, no. 3, September 2004.

[3] Bingchuan Yuan, John Herbert., "Context-aware hybrid reasoning framework for pervasive healthcare," Springer, PersUbiquitComput (2014) 18:865-881.

[4] FemkeOngenae, Maxim Claeys, Thomas Dupont, WannesKerckhove, Piet Verhoeve, Tom Dhaene, Filip De Turck., "A probabilistic ontology-based platform for self-learning context-aware healthcare applications," Elsevier, Expert Systems with Applications 40 (2013) 7629-7646.
[5] Chi-Chun Lo, Chi-Hua Chen, Ding-Yuan Cheng, HsuYang Kung., "Ubiquitous Healthcare Service System with Context-awareness Capability: Design and Implementation," Elsevier, Expert Systems with Applications 38 (2011) 4416-4436.

[6] Yuan B, Herbert J., "Non-intrusive movement detection in cara pervasive healthcare application". In: The 2011 international conference on wireless networks, WORLDCOMP, 2011, pp 360-366.

[7] Hossein Shamsa, Kamran Zamanifar., "MVCC: An Architectural Pattern for Developing Context-aware framework", Elsevier, Procedia Computer Science 34 ( 2014 ) $344-351$.

[8] Yuan B, Herbert J., "Fuzzy CARA—a fuzzy-based context reasoning system for pervasive healthcare". Procedia ComputSci, 2012, 10:357-365.

[9] Bingchuan Yuan, John Herbert, "Transparency Issues in a Hybrid Reasoning Architecture for Assistive Healthcare", Elsevier, AASRI Procedia 4 ( 2013 ) 268 $-274$.

[10] Baldauf M, Dustdar S, Rosenberg F. A survey on context-aware systems. International Journal of Ad Hoc and Ubiquitous Computing. 2007;2(4):263-77.

[11] Hu, B., Hu, B., Wan, J., Dennis, M., Chen, H.-H., Li, L., et al. (2010). Ontology-basedubiquitous monitoring and treatment against depression. Wireless Communications \& Mobile Computing, 10(10), 1303-1319.

[12] B. Yuan, John Herbert, ” Web-based Real-time Remote Monitoring for Pervasive Healthcare", Pervasive Computing and Communications Workshops, IEEE International Conference on (2011), pp.625-629. 